\title{
EXPRESSIVENESS OF METRIC MODALITIES FOR CONTINUOUS TIME
}

\author{
YORAM HIRSHFELD $^{a}$ AND ALEXANDER RABINOVICH ${ }^{b}$ \\ ${ }^{a, b}$ Sackler Faculty of Exact Sciences, Tel Aviv University, Israel 69978. \\ e-mail address: \{joram,rabinoa\}@post.tau.ac.il
}

\begin{abstract}
We prove a conjecture by A. Pnueli and strengthen it showing a sequence of "counting modalities" none of which is expressible in the temporal logic generated by the previous modalities, over the real line, or over the positive reals. Moreover, there is no finite temporal logic that can express all of them over the real line, so that no finite metric temporal logic is expressively complete.
\end{abstract}

\section{INTRODUCTION}

Temporal Logic based on the two modalities "Since" and "Until" $(T L)$ is a most popular framework for reasoning about the evolving of a system in time. By Kamp's theorem [13] this logic has the same expressive power as the monadic first order predicate logic. Therefore the choice between monadic logic and temporal logic is merely a matter of personal preference.

For discrete time these logics suffice. A properties like "Every $X$ will be followed promptly enough by a $Y$ " can be explicitly written once a number $k$ is chosen, and "promptly enough" is interpreted as: "Within $k$ steps".

Temporal logic and the monadic logic are equivalent whether the system evolves in discrete steps or in continuous time. But for continuous time both logics lack the power to express properties of the kind just described, and we must strengthen their expressive power.

Some measure of length of time needs to be included, and the language must be adapted to it. This is done by assuming that there is a basic unit of length, call it "length 1". For predicate logic it is standard procedure to extend the language by a name for the "+1" function, or for a corresponding relation. It will then be the question which fragment of the extended language suits our needs.

Extending temporal logic, without relating it to a corresponding predicate logic, depended on the inventiveness and imagination of the researchers, who created an abundance of approaches and notions, in the work of A. Pnueli, R. Koymans, T. Henzinger and others, 15, 3, 2, 14, 5, 18, 1, 7, 8, and more. Following much work, mainly by Henzinger and collaborators, most of these approaches seem to converge to equivalent languages.

2000 ACM Subject Classification: F.3.1, F.4, F.4.1.

Key words and phrases: Temporal Logics, Expressive completeness, Real Time. 
We evaluated the situation in [10, 12, when most of the work cited was already done, and analyzed the temporal logic in relation to predicate logic. This lead to the temporal logic QTL (Quantitative Temporal Logic), which has besides the modalities Until and Since two metric modalities: $\nabla_{1}(X)$ and $\overleftarrow{\diamond}_{1}(X)$. The first one says that $X$ will happen (at least once) within the next unit of time, and the second says that $X$ happened within the last unit of time. We showed that this logic is equivalent to the different metric temporal logics that we found in the literature, like $M I T L, E C L$ etc. [2, 1, 17, 8. We will use in this paper the logic $Q T L$ as the basic metric temporal logic, but the reader who is acquainted with $M I T L$ or any other of the different metric temporal logics should remember that they are equivalent to $Q T L$ in expressive power, so that the results in this work apply to $M I T L$ and those other languages just as well.

Adding the power to say " $X$ will be true (at least once) within the next unit of time" is natural and necessary. There is however no reason to believe that this gives us the required expressive power. Is it enough, or do we need to add more modalities? If we must add more, which ones should we choose?

A. Pnueli was the first to address these questions. He suggested the modality $P_{2}(X, Y)$ : " $X$ and then $Y$ will both occur in the next unit of time". Pnueli conjectured that the modality $P_{2}(X, Y)$ could not be expressed in $M I T L$ and similar logics (we were unable to locate where this conjecture was first published. It is attributed to Pnueli in later papers like [2] and [18]).

$P_{2}(X, Y)$ was probably thought of as a natural strengthening of the simple metric temporal logics. It can serve as a first in a sequence of extensions of the logic, where for each natural number $n$, we add the modality $P_{n}\left(X_{1}, \ldots, X_{n}\right) . P_{n}\left(X_{1}, \ldots, X_{n}\right)$ says that there is an increasing sequence of points $t_{1}, \ldots, t_{n}$ in the coming unit interval such that $X_{i}\left(t_{i}\right)$ holds for $i=1, \ldots, n$. We call these modalities Pnueli's modalities.

In this paper we will:

- Prove Pnueli's conjecture, that $P_{2}(X, Y)$ cannot be expressed in QTL or MITL.

- Show that none of the modalities $P_{n}\left(X_{1}, \ldots, X_{n}\right)$ can be expressed in terms of the modalities $P_{n-1}\left(X_{1}, \ldots, X_{n-1}\right)$, so that we have a strict hierarchy of modalities.

- And the main result: No temporal logic with a finite set of modalities can express all the modalities $P_{n}\left(X_{1}, \ldots, X_{n}\right)$.

This makes clear two points: That an extension of the simple temporal logics is necessary, and that it will not be as simple as it was for plain temporal logic. It will require infinitely many modalities, and the proper choice is an intriguing question. We hope to address it soon. Note that in predicate logic the expressive power of formulas grows with the increasing of their quantifier depth. In temporal logic there are no quantifiers, and formulas become more complex due to increase in the nesting depth of the modalities that they mention. Kamp showed that for the simplest logic of order iterating the modal operations can replace the complex use of quantifiers. Our result, together with previous evidence (see [16]) suggests that this was a lucky peculiarity of the first-order monadic logic of linear order, and that it cannot be expected to hold for stronger logics.

The main result, that no finite temporal logic can be complete, is not an exact formal claim, until we specify which source of modalities we have in mind. We will specify a natural extensive monadic logic of order, that includes the " +1 " function in its vocabulary. The formal claim will concern the modalities which are definable in this logic. When stated formally the result seems even stronger, as it states not just the incompleteness of temporal 
logics with finitely many modalities, but also of logics with infinitely many modalities, which are defined using bounded quantifier depth.

To state the formal result we define the counting modalities $C_{n}(X)$ which are a simple instance of Pnueli's modalities. $C_{n}(X)$ says that $X$ will hold at least at $n$ points within the next unit of time. $C_{n}(X)$ is a simple instance of the Pnueli modality $P_{n}(X, \ldots, X)$. Our main theorem is the following:

- Let $L$ be second order monadic logic of order, together with the predicate $B(t, s)$ which says that $s=t+1$. The modalities $C_{n}(X)$ are expressible in this logic, but no temporal logic with a finite or infinite family of modalities which are defined by formulas with bounded quantifier depth can express all the modalities $C_{n}(X)$, over the full real line $R$.

Remark 1. Note that our proof applies only to the entire real line. We conjecture that the same claim holds for the positive real line $R^{+}$, but the attempts to prove it became too cumbersome to carry on.

It is well known that in the theory of order, to express the fact that there is a large number of points with a given property, requires formulas with large quantifier depth. We emphasize that the main theorem is not of this nature. Thus in pure temporal logic the two modalities "Until" and "Since" suffice to express for every $n$ the fact that there are $n$ points in the future that satisfy $X$. Less trivial but true, is the fact that in $Q T L$ with its four modalities, for every $n$ there is a formula that says about a point $t$ that $X$ will be true for the length of the interval $(t+n, t+n+1)$ [11]. The nesting of the modalities does for the temporal formulas what the quantifier depth does for the predicate logic formulas. The theorem says that in the general case modality nesting is strictly weaker than quantifier depth, and that no temporal logic will be expressive enough unless it has infinitely many modalities, defined using definitions of unbounded complexity, in terms of quantifier depth.

The paper is divided as follows: In section 2 we recall the definitions and the previous results concerning the continuous time logics. In section 3 we prove Pnueli's conjecture and its generalization, that the modalities $C_{i}$ create a strictly increasing family of logics. In section 4 we discuss the more general and abstract result: that no temporal logic based on modalities with finite quantifier depth can express all the modalities $C_{n}$.

\section{Monadic Logic and Quantitative Temporal Logic}

2.1. MLO - Monadic Logic of Order. The natural way to discuss systems that evolve in time is classical predicate logic. The language has a name for the order relation of the time line, and a supply of unary predicate names to denote a properties that the system may or may not have at any point in time. Hence:

The syntax of the monadic predicate logic of order - MLO has in its vocabulary individual (first order) variables $t_{0}, t_{1}, \ldots$, monadic predicate variables $X_{0}, X_{1}, \ldots$, and one binary relation $<$ (the order). Atomic formulas are of the form $X(t), t_{1}=t_{2}$ and $t_{1}<t_{2}$. Well formed formulas of the monadic logic $M L O$ are obtained from atomic formulas using Boolean connectives $\neg, \vee, \wedge, \rightarrow$ and the (first order) quantifiers $\exists t$ and $\forall t$ and the (second-order) quantifiers $\exists X$ and $\forall X$. The formulas which do not use $\exists X$ and $\forall X$ are called first-order $M L O$ formulas $(F O M L O)$. Note that FOMLO formulas may contain free monadic predicate variables, and they will be assigned to particular predicates in a structure. 
A structure for $M L O$ is a tuple $M=\left\langle A,<, P_{1}, \ldots, P_{n}\right\rangle$, where $A$ is a set linearly ordered by the relation $<$, and $P_{1}, \cdots, P_{n}$, are one-place predicates (sets) that correspond to the predicate names in the logic. We shall use the simple notation $\langle A,<\rangle$ when the particular predicates are not essential to the discussion.

The main models are: the continuous canonical model $\left\langle R^{+},<\right\rangle$, the non-negative real line, and the discrete canonical model $\langle N,<\rangle$, the naturals.

As is common we will use the assigned formal names to refer to objects in the meta discussion. Thus we will write:

$$
M \models \varphi\left[t_{1}, \ldots, t_{k} ; X_{1}, \ldots, X_{m}\right]
$$

where $M$ is a structure, $\varphi$ a formula, $t_{1}, \cdots, t_{k}$ elements of $M$ and $X_{1}, \ldots, X_{m}$ predicates in $M$, instead of the correct but tedious form:

$$
M, \tau_{1}, \ldots, \tau_{k} ; P_{1}, \ldots, P_{m} \models_{\bar{M}_{L O}} \varphi\left(t_{1}, \ldots, t_{k} ; X_{1}, \ldots, X_{m}\right),
$$

where $\tau_{1}, \ldots, \tau_{k}$ and $P_{1} \cdots, P_{m}$ are names in the metalanguage for elements and predicates in $M$.

2.2. Temporal Logics. Temporal logics evolved in philosophical logic and were enthusiastically embraced by a large body of computer scientists. It uses logical constructs called "modalities" to create a language that is free from variables and quantifiers. Here is the general logical framework to define temporal logics:

The syntax of the Temporal Logic $T L\left(O_{1}^{\left(k_{1}\right)}, \ldots, O_{n}^{\left(k_{n}\right)}, \ldots\right)$ has in its vocabulary monadic predicate names $P_{1}, P_{2}, \ldots$ and a sequence of modality names with prescribed arity, $O_{1}^{\left(k_{1}\right)}, \ldots, O_{n}^{\left(k_{n}\right)}, \ldots$ (the arity notation is usually omitted). The formulas of this temporal logic are given by the grammar:

$$
\varphi::=\text { True }|P| \neg \varphi|\varphi \wedge \varphi| O^{(k)}\left(\varphi_{1}, \cdots, \varphi_{k}\right)
$$

A temporal logic with a finite set of modalities is called a finite (base) temporal logic.

Structures for TL are again linear orders equipped with monadic predicates $M=$ $\left\langle A,<, P_{1}, P_{2}, \ldots, P_{n}\right\rangle$, where the predicate $P_{i}$ are those which are mentioned in the formulas of the logic. Every modality $O^{(k)}$ is interpreted in every structure $M$ as an operator $O_{M}^{(k)}$ : $[\mathbb{P}(A)]^{k} \rightarrow \mathbb{P}(A)$ which assigns "the set of points where $O^{(k)}\left[S_{1}, \ldots, S_{k}\right]$ holds" to the $k$-tuple $\left\langle S_{1}, \ldots, S_{k}\right\rangle \in \mathbb{P}(A)^{k}$. (Here $\mathbb{P}$ is the power set notation, and $\mathbb{P}(A)$ denotes the set of all subsets of $A$.) Once every modality corresponds to an operator the semantics is defined by structural induction:

- for atomic formulas: $\langle M, t\rangle \models_{T} P$ iff $t \in P$.

- for Boolean combinations the definition is the usual one.

- for $O^{(k)}\left(\varphi_{1}, \cdots, \varphi_{k}\right)$

$$
\langle M, t\rangle \models_{T L} O^{(k)}\left(\varphi_{1}, \cdots, \varphi_{k}\right) \quad \text { iff } \quad t \in O_{M}^{(k)}\left(A_{\varphi_{1}}, \cdots, A_{\varphi_{k}}\right)
$$

where $A_{\varphi}=\left\{\tau:\left.\langle M, \tau\rangle\right|_{T} L\right\}$ (we suppressed predicate parameters that may occur in the formulas).

We are interested in a more restricted case; for the modality to be of interest the operator $O^{(k)}$ should reflect some intended connection between the sets $A_{\varphi_{i}}$ of points satisfying $\varphi_{i}$ and the set of points $O\left[A_{\varphi_{1}}, \ldots, A_{\varphi_{k}}\right]$. The intended meaning is usually given by a formula in an appropriate predicate logic: 
Truth Tables: A formula $\bar{O}\left(t_{0}, X_{1}, \ldots X_{k}\right)$ in the predicate logic $L$ is a Truth Table for the modality $O^{(k)}$ if for every structure $M$

$$
O_{M}\left(A_{1}, \ldots, A_{k}\right)=\left\{\tau: M \models_{\bar{M}_{L O}} \bar{O}\left[\tau, A_{1}, \ldots, A_{k}\right]\right\} .
$$

The modalities until and since are most commonly used in temporal logic for computer science. They are defined through the following truth tables:

- The modality $X \mathbf{U} Y$, " $X$ until $Y$ ", is defined by

$$
\psi\left(t_{0}, X, Y\right) \equiv \exists t_{1}\left(t_{0}<t_{1} \wedge Y\left(t_{1}\right) \wedge \forall t\left(t_{0}<t<t_{1} \rightarrow X(t)\right)\right) .
$$

- The modality $X \mathbf{S} Y$, " $X$ since $Y$ ", is defined by

$$
\psi\left(t_{0}, X, Y\right) \equiv \exists t_{1}\left(t_{0}>t_{1} \wedge Y\left(t_{1}\right) \wedge \forall t\left(t_{1}<t<t_{0} \rightarrow X(t)\right)\right) .
$$

If the modalities of a temporal logic have truth tables in a predicate logic then the temporal logic is equivalent to a fragment of the predicate logic. Formally:

Proposition 2. If every modality in the temporal logic TL has a truth table in the logic $M L O$ then to every formula $\varphi\left(X_{1}, \ldots, X_{n}\right)$ of TL there corresponds effectively (and naturally) a formula $\bar{\varphi}\left(t_{0}, X_{1}, \ldots X_{n}\right)$ of $M L O$ such that for every $M, \tau \in M$ and predicates $P_{1}, \ldots, P_{n}$

$$
\left\langle M, \tau, P_{1}, \ldots, P_{n}\right\rangle \models_{T} \varphi \quad \text { iff } \quad\left\langle M, \tau, P_{1}, \ldots, P_{n}\right\rangle \models_{M L O} \bar{\varphi} .
$$

In particular the temporal logic $T L(\mathbf{U}, \mathbf{S})$ with the modalities "until" and "since" corresponds to a fragment of first-order $M L O$ (FOMLO).

The two modalities $\mathbf{U}$ and $\mathbf{S}$ are also enough to express all the formulas of first-order $M L O$ with one free variable:

Theorem 3. ([13, 6]) The temporal logic $T L(\mathbf{U}, \mathbf{S})$ is expressively complete for FOMLO over the two canonical structures: For every formula of FOMLO with at most one free variable, there is a formula of $T L(\mathbf{U}, \mathbf{S})$, such that the two formulas are equivalent to each other, over the positive integers (discrete time) and over the positive real line (continuous time).

2.3. QTL - Quantitative Temporal Logic. The logics $M L O$ and $T L(\mathbf{U}, \mathbf{S})$ are not suitable to deal with quantitative statements like " $X$ will occur within one unit of time". In [9, 10, 11] we introduced the Quantitative Temporal Logic, adding to TL the modalities $\diamond_{1} X$ ( $X$ will happen within the next unit of time) and $\overleftarrow{\diamond}_{1} X(X$ happened within the last unit of time):

Definition 4 (Quantitative Temporal Logic). QTL, quantitative temporal logic is the logic $T L(\mathbf{U}, \mathbf{S})$ enhanced by the two modalities: $\nabla_{1} X$ and $\overleftarrow{\diamond}_{1} X$. These modalities are defined by the tables with free variable $t_{0}$ :

$$
\begin{array}{ll}
\diamond_{1} X: & \exists t\left(\left(t_{0}<t<t_{0}+1\right) \wedge X(t)\right) \\
\overleftarrow{\diamond}_{1} X: & \exists t\left(\left(t<t_{0}<t+1\right) \wedge X(t)\right) .
\end{array}
$$

$Q T L$ was the latest in a list of metric logics for continuous time, developed over approximately 15 years. When interpreted carefully all these logics are equivalent. We refer the reader to [15, 3, 2, 14, 15, 18, 1, 7, 8] for some of the previous work. 
The novelty in our approach was the close connection with metric monadic logic, the replacement of all the automata theory arguments by plain logic and model theory arguments. Most significant however was the fact that our treatment and our results applied uniformly to the class of systems with finite variability and to the class of all systems. In contrast, in the previous work, and in particular in the papers cited above, systems without finite variability could not be defined as the semantics for the logic. Naturally the decidability and complexity results did not apply to systems without finite variability (nor could the automata approach be adapted to the general case once the definition includes general systems).

We proved in [10] and [12] that:

(1) QTL consumes the different decidable metric temporal logics that we found in the literature, including $M I T L, E C L$ etc.

(2) There is a natural fragment $Q M L O$ (quantitative monadic logic of order), of the classical monadic logic of order with the +1 function, that is equal in expressive power to QTL.

(3) The validity and satisfiability problem for this logic is decidable, whether we are interested in systems with finite variability, or in all systems evolving in time (a system has finite variability if it changes only at finitely many points, in any finite interval of time).

For the special case of systems with finite variability these results (but not the proof methods) are in [15, 3, 2, 14, 5, 18, 1, 7, 8, regarding different logics (future, or full), and different semantics (point sequence and interval sequence).

\section{Modalities Which ARE NOT EXPRESSible IN $Q T L$}

The simple metric temporal logic $Q T L$ looks very natural. The main question is if it is as expressive as is needed. A. Pnueli suggested a natural modality, and conjectured that it could not be expressed in the simple metric temporal logics of the previous section. This was the modality that we denote by $P_{2}(X, Y)$, which says that $X$ and then $Y$ will be true at two points in the next unit of time. If the conjecture is confirmed then we have a natural modality to add to the logic. Moreover, there are $P_{3}(X, Y, Z)$ and $P_{n}\left(X_{1}, \ldots, X_{n}\right)$ waiting to be considered as an addition, if they are not redundant.

We attend these questions and we will prove first Pnueli's conjecture, that $P_{2}(X, Y)$ is not expressible in $Q T L$, and then that there is a proper hierarchy of Pnueli modalities that can be added to strengthen the logic.

\section{Definition 5.}

(1) The counting modalities are the modalities $C_{n}(X)$ for every $n$ which state that $X$ will be true at least at $n$ points within the next unit of time.

(2) The Pnueli modalities are the modalities $P_{n}\left(X_{1}, \ldots, X_{n}\right)$ for every $n$ which state that there is an increasing sequence of points $t_{1}, \ldots, t_{n}$ in the unit interval ahead, such that for $i=1, \ldots, n, t_{i}$ is in $X_{i}$.

Pnueli's conjecture is proved by the following theorem:

Theorem 6. The modality $C_{2}(X)$ is not expressible in $Q T L$.

Proof. Let $M$ be the real non negative line with the predicate $P(t)$ that is true exactly at the points $n \cdot \frac{2}{3}$ for all natural numbers n. Let us call the following four predicates: $P, \neg P$, True, False the trivial predicates. We show by structural induction that for every 
statement $\varphi$ of $Q T L$ there is a point $t_{\varphi}$ such that from this point on $\varphi$ is equivalent to one of the trivial predicates.

- this is trivially true for atomic statements.

- The collection of truth sets for the four trivial predicates is closed under Boolean combinations. Therefore the set of formulas satisfying our claim is closed under the Boolean connectors.

- Assume now that $\varphi=(\theta \mathbf{U} \psi)$ and $t_{0}$ is a point beyond which both $\theta$ and $\psi$ are equivalent to one of the trivial predicates. We check the different possibilities for the truth value of $\varphi$ at a point $t$ beyond $t_{0}$. If $\theta$ is equivalent to $P$ or to False then $\varphi$ is false. If $\theta$ is equivalent to $\neg P$ or to True then $\varphi$ is true if $\psi$ is equivalent to either of $P, \neg P$ or True, and $\varphi$ is false if $\psi$ is equivalent to False. In every case $\varphi$ is equivalent either to True or to False.

- For $\varphi=(\theta$ Since $\psi)$ we need only a minor modification: Let $t_{1}$ be an even integer beyond $t_{0}$ (so that $P$ is true at $t_{1}$ ). Then for points beyond $t_{1} \varphi$ is true if $\theta \equiv$ True and $\psi$ occurred at $t_{1}$ or earlier, or if $\theta \equiv \neg P$ and $\psi$ is equivalent to any of the special predicates except False (the choice of $t_{1}$ ensures the case that $\psi \equiv P$ ) in all other cases $\varphi \equiv$ False.

- Assume that $\varphi=\nabla_{1} \theta$ and from $t_{0}$ on $\theta$ is equivalent to one of the four trivial predicates. If $\theta$ is equivalent to False then $\varphi$ is equivalent to False from $t_{0}$ on. In the other three cases $\varphi$ is equivalent to True from $t_{0}$ on.

- A similar argument works when $\varphi=\overleftarrow{\diamond}_{1} \theta$.

On the other hand the statement $C_{2}(P)$ is false at any point in the interval $(n, n+1 / 3)$ if $n$ is even and it is true at any point in the interval $(n, n+1 / 3)$ if $n$ is odd. This shows that $C_{2}(P)$ is not equivalent to any $Q T L$ formula.

The method of the proof can be adapted to show that the Pnueli modalities yield a strictly monotone sequence of temporal logics:

Theorem 7. The modality $C_{n}(X)$ is not expressible in the logic $Q T L\left(P_{2}, \cdots, P_{n-1}\right)$.

Proof. Let $M$ be the real non negative line with the predicate $P(t)$ that is true exactly at the points $k \cdot \frac{2}{2 n-1}$ for all natural numbers k. Call again the following four predicates: $P, \neg P$, True, False the trivial predicates, and as before show that every formula of $\operatorname{QTL}\left(P_{2} \cdots, P_{n-1}\right)$ is equivalent from some point on to a trivial predicate. The proof remains the same except for the additional induction step, where we assume that the claim is true for $\varphi_{1}, \ldots, \varphi_{n-1}$. and we must show that it holds for $\psi=P_{n-1}\left(\varphi_{1}, \ldots, \varphi_{n-1}\right)$. By assumption there is some point from which on $\varphi_{1}, \ldots, \varphi_{n-1}$ are trivial. If any of them is False then $\psi$ is false from there on. Otherwise $\psi$ is True from there on, because at any point there are $n-1$ points of $P$ in the future unit, and between any two, there are infinitely many points that satisfy $\neg P$ or True.

On the other hand $C_{n}(P)$ is always true on the interval $\left(k, k+\frac{1}{2 n-1}\right)$ if $k$ is even, and false on the interval if $k$ is odd.

Remark 8. We proved the two theorems for the positive real line. I.e, for continuous time with a first moment. The same proof applies to the full real line, to the set of rational numbers or to the set of positive rational numbers. 


\section{The INCOMPLETENESS OF TEMPORAL LOGIC WITH FINITELY MANY MODALitieS}

The hierarchy

$$
T L<Q T L<Q T L\left(P_{2}\right)<\cdots<Q T L\left(P_{2}, \cdots, P_{n}\right)<\cdots
$$

raises the suspicion that it will be difficult to find a finite temporal logic that includes all these logics. In this section we will prove that it is indeed impossible. To be precise:

Theorem 9. Let $L$ be the second order monadic logic of order, with an extra predicate $B(t, s)$ that is interpreted on the whole real line as $s=t+1$. Let $L_{1}$ be a temporal logic with possibly infinitely many modalities, for which there is a natural number $m$ such that all the modalities have truth tables in $L$, with quantifier depth not larger than $m$. Then there is some $n$ such that $C_{n}(X)$ is not equivalent over the real line to any $L_{1}$ formula.

Before we start to work toward the proof we make the following remarks.

(1) Second order monadic logic of order with the +1 function is a much stronger logic than is usually considered when temporal logics are defined. All the temporal logics that we saw in the literature are defined in a fragment of monadic logic, with a very restricted use of the +1 function. All the decidable temporal logics in the literature remain decidable when we add the counting modalities $C_{n}(X)$ [11. On the other hand second order monadic logic is undecidable over the reals even without the +1 function [17. When the +1 function is added even a very restricted fragment of first order monadic logic of order is undecidable over the positive reals.

(2) The theorem says that there is no finite set of modalities defined in this language that generates all the counting modalities (and possibly more). It does not exclude the possibility that a finite set of modalities which are not defined in this logic is (at least) as strong as $Q T L\left(P_{2}, \ldots, P_{n}, \ldots\right)$. We state it as an open problem:

Question: Is there a finite temporal logic that includes all the modalities $P_{n}\left(X_{1}, \ldots, X_{n}\right)$, if we do not require that the modalities are are defined by truth tables?

(3) On the other hand the theorem does not just say that $Q T L\left(P_{2}, \ldots, P_{n}, \ldots\right)$ is not a sublogic of a logic with finitely many modalities. It is not even a sublogic of an infinite temporal logic whose modalities are defined with bounded quantifier depth, in a strong predicate logic.

The proof of theorem 9 involves some more notations, and some steps that are accumulated in two more theorems.

We will assume that $P$ is the only non variable unary predicate name in $L$, and we concentrate on a class of simple models in the language: For each integer $k>0$ let $M_{k}$ be the full real line $R$ with $P(t)$ occurring at the points $m \frac{1}{k}$ for every integer $m$ (positive, negative or zero).

\section{Terminology.}

(1) From now on whenever we say "a model" we mean $M_{k}$ for some $k$.

(2) The four formulas $\{$ True, False, $P, \neg P\}$ will be called the trivial temporal logic formulas, and the formulas $\left\{\right.$ True, False, $\left.P\left(t_{0}\right), \neg P\left(t_{0}\right)\right\}$ will be called the trivial first-order formulas.

(3) We say that $t$ is a $P$-point or that $t$ is in $P$ if $P(t)$ is true.

The choice of the models limits the expressive power of monadic logic: 
Theorem 10. Every formula of second order monadic logic of order, with the unary predicate $P$ and with the extra predicate $B(t, s)$ with one free element variable $t_{0}$ and no free predicate variable is equivalent in every model to one of the four trivial formulas.

Proof. Let $M_{k}$ be given. We show that for every two points $t<s$ in $P$, there is an automorphism of the model that maps $t$ to $s$ and for every two points not in $P$ there is such an automorphism. This will prove that every formula obtains the same truth value on all points in $P$ and the same truth value on all points not in $P$. Therefore the formula is true either everywhere, or only on points in $P$, or only outside of $P$, or nowhere.

(1) the mapping $G(t)=t+r$ is an automorphism if $r$ is a multiple of $\frac{1}{k}$. This shows in particular that every formula obtains a fixed truth value on all points in $P$.

(2) For every $0<t<s<\frac{1}{k}$ there is a monotone bijection $h(v):\left(0, \frac{1}{k}\right) \rightarrow\left(0, \frac{1}{k}\right)$ such that $h(t)=s$. Every real $t$ can be written in a unique way as $t=\frac{m}{k}+\tau$ where $m$ is integer and $0 \leq \tau<\frac{1}{k}$, and the bijection extends to an automorphism of the model defining $H\left(\frac{m}{k}+\tau\right)=h(\tau)+\frac{m}{k}$. This shows that every formula obtains the same truth value on the interval $\left(0, \frac{1}{k}\right)$.

(3) Finally if $t=\frac{m}{k}+\tau$ where $m$ is integer and $0 \leq \tau<\frac{1}{k}$ then every formula has the same truth value on $t$ and on $\tau$, so that it is fixed on the complement of $P$.

Thus every formula is equivalent to one of the four trivial formulas in every model. Note that it is not necessarily the same trivial formula in the different models. For example $C_{k}(P)$ is equivalent to $\neg P$ in $M_{k}$ and to True in $M_{k+1}$. We aim to show that in a temporal logic based on modalities defined by formulas of bounded quantifier depth, there are always pairs of models for which any formula is equivalent in both to the same trivial formula.

We denote by $L_{n}$ the set of formulas of quantifier depthes no more than $n$ of the second order monadic logic of order with the extra predicate $B(t, s)$. We denote by $T L_{n}$ the temporal logic with all the modalities that have a truth table in $L_{n}$. Every formula of $T L_{n}$ is equivalent in every model to one of the trivial formulas.

Let now $\varphi\left(t_{0}, X_{1}, \ldots, X_{k}\right)$ be a formula of $L_{n}$. Its localizations are the $4^{k}$ formulas $\varphi\left(t_{0}, T_{1}, \ldots, T_{k}\right)$, where $T_{1}, \ldots, T_{k}$ vary over all possible combinations of trivial predicates $P, \neg P$, True, False. Note that the localizations have the same quantifier depth as the original formula, and that beside the predicate name $P$ there are no predicate variables in the localizations.

Here is the main theorem. Item (4) is the promised result:

Theorem 11. Let $n$ be given and let $S_{n}$ be the collection of the localizations of all the (truth tables of) modalities in $T L_{n}$. Then:

(1) Let $M=M_{k}$ and $M^{\prime}=M_{l}$ be models. If there is a formula of $T L_{n}$ which is equivalent to different trivial formulas in $M$ and in $M^{\prime}$ then there is also such a formula in $S_{n}$.

(2) There are finitely many formulas in $S_{n}$ such that every formula of $S_{n}$ is logically equivalent to one of them.

(3) There are models $M_{k}$ and $M_{l}$ for which any formula of $T L_{n}$ is equivalent to the same trivial formula in both models.

(4) There are integers $k$ such that $C_{k}(X)$ is not expressible in $T L_{n}$.

Proof.

(1) We assume that every formula in $S_{n}$ is equivalent to the same trivial formula in $M$ and in $M^{\prime}$ and we prove by structural induction that the same is true for every formula 
$\varphi$ of $T L_{n}$. If $\varphi$ s atomic it is $P$ or True. If it is a Boolean combination of simpler formulas then the property is trivially inherited from the simpler formulas. It remains to check the case where $\varphi=O^{(k)}\left(\varphi_{1}, \cdots, \varphi_{k}\right)$. By assumption for $i=1 \cdots k, \varphi_{i}$ is equivalent in both models to the same trivial formula $T_{i}$. If now $\bar{O}\left(t_{0}, X_{1}, \ldots X_{k}\right)$ is the truth table for the modality $O^{(k)}\left(X_{1}, \cdots, X_{k}\right)$ then $\varphi$ is equivalent in both models to $\bar{O}\left(t_{0}, T_{1}, \ldots T_{k}\right)$. This is a formula in $S_{n}$ and therefore expresses the same trivial predicate in both models.

(2) This is a well known simple property of logics with finite relational signature: For every $n$ and $m$ there are finitely many quantifier free formulas such that every quantifier free formula with variables among $v_{1}, \cdots, v_{n}$ and $Y_{1}, \cdots, Y_{m}$, is logically equivalent to one of them. Consequently for every $q, m$ and $n$ there is a finite number of formulas such that every formula of quantifier depth $n$ (of both first and second order variables) and in the free variables $v_{1}, \cdots, v_{q}$ and $Y_{1}, \cdots, Y_{m}$, is logically equivalent to one of them. $S_{n}$ is a special case where $q=1$ and $m=0$.

(3) Let $\phi_{1}, \ldots, \phi_{r}$ be a list of formulas such that every formula of $S_{n}$ is logically equivalent to one of the formulas in the list. We partition the class of models into $4^{r}$ classes according to which trivial predicate is defined by these $r$ formulas. Then at least one class is infinite. Every formula in $S_{n}$ is equivalent in all the models in this class to the same trivial formula. By (1) any formula of $T L_{n}$ is equivalent to the same trivial predicate in all models in this class.

(4) Let now $M_{k}$ and $M_{l}$ be in this infinite class with $k<l . C_{k}(P)$ is equivalent to $\neg P$ in $M_{k}$ and to True in $M_{l}$. However, by (3) and our choice of $k$ and $l$, every formula of $T L_{n}$ is equivalent to the same trivial formula in both models $M_{k}$ and $M_{l}$. This shows that $C_{k}$ is not equivalent to any $T L_{n}$ formula.

The proof of theorem 9 is just item 4 in theorem 11 .

Some remarks are in order:

(1) The theorem speaks about the whole real line, and not about its non negative part $R^{+}$. We believe that it is true also for the model of non negative reals, but we did not pursue the proof, which is complicated by everything that can be said about 0 , and therefore about every particular $n$, and about every particular interval $(n, n+1)$. We state it as a question:

Question: Is the theorem above true when the real line $R$ is replaced by its non negative part $R^{+}$?

(2) The completeness of the real line was never used and the same proof works for the model of the rational numbers.

(3) Adding just a " +1 " function is weaker than adding the functions " $+q$ " for every rational number $q$. Let us denote by $L_{Q}$ the monadic logic of order with predicates $B_{q}(t, s)$ for every rational umber $q$, to express the relation $s=t+q$. The proof of the theorem will not apply if we replace $L$ by $L_{Q}$, and even modalities with truth tables of quantifier depth 2 distinguish between any two models $M_{k}$ and $M_{r}$ in our class. On the other hand just as before no finite temporal logic defined in this logic can express all the counting modalities. This is the case because any finite number of modalities defined in $L_{Q}$ involves only finitely many rational numbers in the formulas $B_{q}(t, s)$ that occur in the definition. Let $q_{0}$ be a rational such that all of these rational are whole integers of 
$q_{0}$. Then we can repeat the proof above with $q_{0}$ replacing 1 . We do not know whether infinitely many modalities defined with bounded quantifier depth may suffice:

Question: Is the theorem above true when the predicate logic $L$ is replaced by $L_{Q}$ ?

\section{Conclusion}

Temporal logic does not have quantifiers, and formulas become more complex only due to deeper nesting of modalities. By Kamp's theorem this suffices to capture the expressive power that is achieved in predicate logic by quantifier depth, and the pure temporal logic with its two modalities is as expressive as the pure monadic logic of order. We proved that in the more general setting of a metric temporal logic, nesting of modalities is strictly weaker than nesting of quantifiers. Only a set of modalities defined with unbounded quantifier depth can capture all the counting modalities $C_{n}(X)$.

\section{REFERENCES}

[1] R. Alur, T. Feder, T.A. Henzinger. The Benefits of Relaxing Punctuality. Journal of the ACM 43 116-146, (1996).

[2] R. Alur, T.A. Henzinger. Logics and Models of Real Time: a survey. In Real Time: Theory and Practice. Editors de Bakker et al. LNCS 600 74-106, (1992).

[3] H. Barringer, R. Kuiper, A. Pnueli. A really abstract concurrent model and its temporal logic. Proceedings of the 13th annual symposium on principles of programing languages 173-183, (1986).

[4] H.D. Ebbinghaus, J. Flum, Finite Model Theory. Perspectives in mathematical logic, Springer (1991).

[5] D.M. Gabbay, I. Hodkinson, M. Reynolds. Temporal Logics volume 1. Clarendon Press, Oxford (1994).

[6] D.M. Gabbay, A. Pnueli, S. Shelah, J. Stavi. On the Temporal Analysis of Fairness. 7th ACM Symposium on Principles of Programming Languages. Las Vegas 163-173, (1980).

[7] T.A. Henzinger. It's about time: real-time logics reviewed. In Concur 98, Lecture Notes in Computer Science 1466, pp. 439-454, (1998).

[8] T.H Henziger, J.F Raskin, P.Y Schobbens. The regular real time languages. ICALP98, pp. 580-591, (1998).

[9] Y. Hirshfeld and A. Rabinovich, A Framework for Decidable Metrical Logics. In Proc. 26th ICALP Colloquium, LNCS vol.1644, pp. 422-432, Springer Verlag, (1999).

[10] Y. Hirshfeld and A. Rabinovich. Quantitative Temporal Logic. In Computer Science Logic 1999, LNCS vol. 1683, pp. 172-187, Springer Verlag (1999).

[11] Y. Hirshfeld and A. Rabinovich, Logics for Real Time: Decidability and Complexity. Fundam. Inform. 62(1):1-28 (2004).

[12] Y. Hirshfeld and A. Rabinovich, Timer formulas and decidable metric temporal logic. Information and Computation Vol 198(2), pp. 148-178, (2005).

[13] H. Kamp. Tense Logic and the Theory of Linear Order. Ph.D. thesis, University of California L.A. (1968).

[14] Z. Manna, A. Pnueli. Models for reactivity. Acta informatica 30:609-678, (1993).

[15] R. Koymans. Specifying Real-Time Properties with Metric Temporal Logic. Real-Time Systems 2(4):255-299, (1990).

[16] A. Rabinovich. Expressive Power of Temporal Logics In Proc. 13th Int. Conf. on Concurrency Theory, vol. 2421 of Lecture Notes in Computer Science, pp. 57-75. Springer, (2002).

[17] S. Shelah. The monadic theory of order. Ann. of Math., 102, pp. 349-419, (1975).

[18] T. Wilke. Specifying Time State Sequences in Powerful Decidable Logics and Time Automata. In Formal Techniques in Real Time and Fault Tolerance Systems. LNCS 863, pp. 694-715, (1994).

This work is licensed under the Creative Commons Attribution-NoDerivs License. To view a copy of this license, visit http://creativecommons.org/licenses/by-nd/2.0/ or send a letter to Creative Commons, 559 Nathan Abbott Way, Stanford, California 94305, USA. 